\title{
IMPLEMENTATION AND ANALYSIS OF A HANDWRITTEN SIGNATURE VERIFICATION TECHNIQUE
}

\author{
Alan McCabe \\ Fern Computer Services, Pty Ltd. \\ Belfast, Ireland \\ Jarrod Trevathan \\ School of Maths, Physics and Information Technology \\ James Cook University
}

Keywords: Biometric security, authentication, dynamic features, string comparison, extrema avoidence.

\begin{abstract}
There is considerable interest in authentication based on handwritten signature verification because it is superior to many other biometric authentication techniques such as finger prints or retinal patterns, which are reliable but much more intrusive. The paper details a number of experiments using a signature verfication technique which is unlike any other reported in literature. Specifically, characters are used to represent various features of a signature image allowing the use of existing and proven string distance metrics to determine distances between signatures. Extensive testing shows that our proposed system is comparable with, and in many aspects better than the highest quality signature verfication techniques presented in literature.
\end{abstract}

\section{INTRODUCTION}

Biometric security systems such as finger prints and retinal scanning are increasingly being used as a means of authenticating an individual's identity. Despite the reliability of these systems, they are somewhat intrusive and are often met with resistance by those being authenticated. A more natural and acceptable authentication method is to verify an individual's handwritten signature. This involves capturing the signature via a graphics tablet and comparing this with a reference file. The process involved is essentially the same as a credit card transaction, however, the procedure is automated and the ultimate verification decision is made by a computer program rather than relying on human judgment. (See (McCabe, 2000; Trevathan and McCabe, 2005).)

This paper presents a Handwritten Signature Verification (HSV) system which extends an approach described by (Gupta and Joyce, 1997a). Their approach involves abstracting the signature data into a character string representing the more discriminating signature features. This approach's difficulty lies in determining the type of features to capture, how to extract them from the signature data, and how to incorporate the extracted features into the character string.

This paper aims to improve the performance of the techniques developed by (Gupta and Joyce, 1997a) by combining shape and dynamics of the signature in a number of different ways using a single-stage approach. The proposed HSV system's goals include:

1. Uses no more than five reference signatures.

2. Does not have unrealistic resource requirements.

3. Thoroughly tested.

4. Can store reference signature information on a credit card or a smart card.

This paper is organised as follows: Section 2 provides background relating to the approach used by our HSV system. Section 3 presents the methodology behind the proposed HSV system, along with a description of various novel approaches taken to deal with problems encountered. It also evaluates the HSV system's performance and its limitations. Section 4 provides some concluding remarks.

\section{BACKGROUND}

This section describes specific approaches towards solving the HSV problem and the motivation for our proposed HSV system.

HSV involves the following five phases: data $a c$ quisition, preprocessing, feature extraction, comparison process, and performance evaluation. During the 
first three phases most methods would generate a reference signature (or a set of reference signatures) for each individual. This requires a number of user signatures to be captured at enrollment/registration time and processed. In the discussion that follows, it is assumed that there is only one reference signature. When a user claims to be a particular individual, they present a test signature, which is compared with the reference signature for that individual. The difference between the two is then computed using a distance measure. If the distance is above a predefined threshold value, then the test signature is rejected as a forgery, otherwise it is authenticated.

The following notion for performance evaluation is used throughout this paper:

- $r$ : the threshold value. (The threshold is $r$ times the standard deviations plus the reference mean.)

- F-accept: number of forgeries accepted as genuine.

- G-reject: number of genuine signatures rejected as forgeries.

- FAR: false acceptance rate expressed as a percentage.

- FRR: false rejection rate expressed as a percentage.

- ER: total error rate (FAR + FRR).

Performance evaluation of the proposed technique is very important and normally researchers use a set of genuine signatures and forgery attempts collected by them (or by someone else), and determine the FRR and FAR for the technique given the signature database. Obtaining good FAR estimates is very difficult since real-world successful forgeries are impossible to obtain. Performance evaluations rely on two types of forged signatures. A forgery may be skilled, if it is produced by a person other than the individual whose signature is being forged when the forger has had access to one or more genuine signatures for viewing and/or practice. A forgery is called zero effort or random when either another person's genuine signature is used as a forgery, or the forger has no access to the genuine signature and is either only given the name of the person whose signature is to be forged, or just asked to sign any signature without even knowing the name. Tests on random forgeries generally lead to a much smaller FAR than on skilled forgeries.

There are quite a large number of other systems which deal with HSV. However, almost all of them exhibit several shortcomings. This work's main priority is to avoid these shortcomings and produce a system which not only performs well, but is suitable for real world applications. Four such pitfalls include:

Use of Too Many Reference Signatures - there is a direct correlation between the number of reference signatures and the quality of the reference created using those signatures. It is very important not to assume the existence of too many reference signatures, as it may not always be possible to obtain them.

Failure to Combine Static and Dynamic Features - a signature is made up of features which are both static (i.e., shape related such as the length of long strokes) and dynamic (i.e., motion related such as the velocity and pin tip acceleration at various stages).

Unrealistic Resource Requirements - although performing huge amounts of preprocessing and calculations may result in high levels of reliability, it is important that the signature verifier is fast. It is not acceptable for a system user to have to wait a few minutes every time they desire to verify a signature.

Insufficient Testing - although most authors report error rates in the form of both FAR and FRR, the level of testing style varies greatly throughout literature, making comparisons between different systems difficult. A realistic testing level must involve a high number of tests for false rejection (genuine signatures being rejected as forgeries) and false acceptance (forgeries, which should be high in both quality and number, being accepted as genuine signatures). In addition, it is not realistic to remove signatures from testing simply on the basis that they do not work well with the desired system, nor is it realistic to nonrandomly choose signatures to be used as a reference, testing or otherwise. Finally, only one attempt should be allowed for a user signature to be verified - many systems allow a user three attempts to verify a signature and this is generally not acceptable.

There are three main categories which HSV systems can fall into: point-to-point comparison, feature values comparison, and capturing signature shape.

Point-to-Point Comparison compares a test signature with a reference signature by comparing different parts of the signature separately and combining these comparisons to achieve an overall similarity measure. A number of investigations suggest that point-to-point techniques can lead to good results but the techniques suffer from difficulties due to variations in the genuine signatures of each individual. In order to make more effective comparisons, a system must perform some type of alignment of the test and reference signatures in an attempt to "line-up" the corresponding parts of the signatures. This alignment may in turn create problems of its own, since forgeries will undergo alignment as well as genuine signatures. 


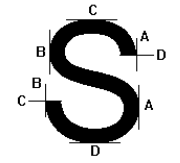

Figure 1: The valley/peak characters associated with the letter 'S'.

It appears that highly reliable HSV systems are unlikely to be based on such techniques. Some low error rates have been reported, but the testing procedure performed on these systems were quite inadequate. The best results so far for well tested systems seem to give total error rates of just over $15 \%$. Also, the systems have not been tested for zero-effort FAR.

Feature Values Comparison is relevant to this paper, because many features are captured implicitly in the representation. For example, the number of turning points, the pen-up time and the total time taken to sign. There are two important issues within this type of approach. Firstly, it is necessary to decide on the number and type of features to capture, and secondly it is important to choose an appropriate means of comparing those feature values.

Most HSV techniques are based on statistical features capturing mainly dynamic details of the signature. The feature-based techniques work remarkably well without having to store the entire signature, but there is a limit to the performance level which can be obtained. Most HSV proposals have error rates of more than $10 \%$ and $15 \%$. These systems have not been tested for a zero-effort FAR.

Capturing Signature Shape - Most static HSV is based on capturing the shape or some aspects of the signature's shape. Dynamic HSV involves capturing details relating to signature dynamics (e.g., pen tip velocity, acceleration, etc.). This category essentially is a combination of static and dynamic HSV in that it uses dynamic information and signature shape.

The (Gupta and Joyce, 1997a) technique assumes that the signature is captured using a device capable of obtaining the $(x, y)$ coordinate pairs several times per second (usually of the order of 200 samples per second). The $x$ and $y$ data is then extracted into separate vectors (known as profiles). The verification technique can be explained using the following symbols:

$\begin{array}{ll}A \text { for a peak of the } x \text { profile } & B \text { for a valley of the } x \text { profile } \\ C \text { for a peak of the } y \text { profile } & D \text { for a valley of the } y \text { profile }\end{array}$

A signature may now be processed to identify all the peaks/valleys in the $x$ and $y$ profiles, and each of the profiles may then be represented by a string of symbols representing the peaks/valleys. The $x$ profile was then represented by a string $A B A B A B A B \ldots$ and the $y$-valley profile by a string like $C D C D C D$ $C D C$... with each peak and valley in the two profiles having a time associated with it (which was not displayed in this simple representation). The peak and valley times may then be used to interleave the $x$ and $y$ profile representations so that the signature shape was represented by a string like $D A C B A D B C$. This simple example indicates that the signature's $x$ and $y$ profiles together had only nine peaks and valleys (typically there might be 50 or more) that were $x$-peak followed by $y$-peak, then an $x$-valley, etc. Another way to look at this representation was to view it as a description of the pen motion, i.e., from the initial position ( $x$ peak) the pen first moved northwest to reach a $y$-peak then southwest to reach an $x$-valley and then turned around and moved southeast to reach an $x$-peak and $y$-valley and so on. $D A C B A D B C$ therefore represents a pen motion something similar to the letter $S$ written from the top to bottom (see Figure 1). The representation would be reversed if the letter $S$ was written from the bottom to the top. The representation does not capture the curvature or the size of the curves that make the letter $S$ and therefore $D A C B A D B C$ was a representation for many curves that look somewhat similar and thus the representation provides considerable flexibility and tolerates considerable variation in the way the pen moves. The representation captures the shape and the direction of pen movement during signature writing. Given the flexible representation, similar representation should always be obtained for a person's signatures in spite of minor variations in the genuine signatures.

A small number of systems have been built using techniques for capturing signature shape in a way that is quite different than the approach used in the point-to-point comparisons. Most shape systems suffer from poor performance when skilled forgeries are tested, since skilled forgers are often able to reproduce the shape of the signature quite well. Simple techniques which capture shape and then compare it therefore are unlikely to produce high performance for skilled forgeries. This is in contrast to the performance of the dynamic feature-based techniques which perform very well when skilled forgeries are tested, since the dynamics of skilled forgeries are often very different than that of the genuine signatures.

\section{IMPLEMENTATION}

This section discusses the basic methodology, design, implementation and performance evaluation of our HSV system. 
The major HSV software components are as follows:

1. Input component: responsible for reading in the signature data from signature files to the program.

2. Preprocessor: detects the genuine valleys/peaks in the signature data read in during input and indexes them according to their position in the signature.

3. String generator: takes the ordered set of valleys and peaks as well as their positions and produces a character string representing the signature data.

4. String comparison: uses the character strings to compare two signatures and determines whether a signature is to be accepted or rejected.

5. Report generator: collates the accepted/rejected signatures and obtains error rates for false acceptance and false rejection. A detailed report for each individual in the database, as well as for the database as a whole is then written to an output file.

The performance evaluation of our proposed HSV system uses the same database employed by (Gupta and Joyce, 1997b). The database contains a total of 1229 signatures taken from 59 users. There are 904 genuine signatures, each user providing usually 15 genuine signatures. There are also 325 highly skilled forgeries, about five for each user. Further details of the database can be found in (Nelson et al, 1994). In most experiments five signatures were used for each reference leaving $904-(5 \times 59)=609$ genuine signatures for computing FRRs.

\section{Experimental Protocol}

An experimental protocol similar to that described in (Gupta and Joyce, 1997b) was used. The protocol remained constant throughout the experimentation.

To create a reference signature, the first five signatures for each user were taken from the database and transformed into strings using the technique being evaluated. The five strings were then compared with each other using a string distance algorithm and the ten distances were obtained. The reference mean and the reference standard deviation of the distances were computed. These five signatures, for each user, are not used again as test signatures.

The remaining genuine/forged signatures for that user are tested against the five reference signatures. This is done by determining the distance between the test signature string and each of the reference strings, and finding the minimum of these distances. If this minimum distance (known as the test distance) is smaller than the threshold distance, the test signature is accepted, otherwise it is rejected.
Various values of threshold were used in performance evaluation; the threshold being the sum of the reference mean and $r$ times the reference standard deviation. The value of $r$ depends on the type of application that the HSV system is to be used for. If security (low FAR) is the major concern, then a small value for $r$ will be used. Alternately, if a low FRR is the major concern, then a larger threshold is used.

\section{Comparing Two Strings}

The importance of selecting and implementing an appropriate algorithm for finding the distance between the two strings should not be underestimated, since performance relies on the string distance algorithm to determine accurately the distance between signatures' string representations. The WagnerFischer (WF) string distance algorithm (Wagner and Fischer, 1974) best suited our purpose.

The WF dynamic-programming method is based upon successively evaluating the distance between longer and longer prefixes of the two strings until the final result is obtained. The partial results are computed iteratively and are entered into an $(m+1) \times$ $(n+1)$ array (where $m$ and $n$ are the lengths of the two strings being compared), leading to $O(m n)$ time and space complexities.

The algorithm involves calculating the cost of transforming one string into another in an iterative fashion. For example, the cost of transforming one string into an empty string to another empty string is obviously zero, the cost of transforming from one empty string with a single character is one. More generally, if the cost of transforming $x(1, i)$ into $y(1, j-$ $1)$ is known, then the cost of transforming $x(1, i)$ into $y(1, j)$ may be obtained by adding the cost of inserting $y_{j}$. Similarly, if the cost of transforming $x(1, i-1)$ into $y(1, j-1)$ is known, then the cost of transforming $x(1, i)$ into $y(1, j)$ may be obtained by adding the cost of replacing $x_{i}$ with $y_{j}$. The primary advantage of the WF algorithm for use in this application is that the string $A B$ can be transformed into the string $B A$ at a cost of only one. It is possible to use the iterative nature of this algorithm to create a matrix where the elements contain the cost of transforming from one substring to another.

For example, suppose there are two character strings $A B C D A$ and $B A D C A$, then the WF matrix looks like:

$\begin{array}{lllllll} & & A & B & C & D & A \\ & 0 & 1 & 2 & 3 & 4 & 5 \\ B & 1 & 1 & 1 & 2 & 3 & 4 \\ A & 2 & 1 & 2 & 2 & 3 & 3 \\ D & 3 & 2 & 2 & 3 & 2 & 3 \\ C & 4 & 3 & 3 & 2 & 3 & 3 \\ A & 5 & 4 & 4 & 3 & 3 & 3\end{array}$


The overall distance between the two strings $A B C D A$ and $B A D C A$ is 3 .

\section{Valley and Peak Detection Algorithm}

A simple valley and peak detection algorithm would involve detecting a valley (and similarly a peak), if there was a 'down' motion, followed possibly by some number of 'flats' (possibly zero), followed by an 'up' motion. For example, suppose the following data existed in the $y$ direction:

...,102,101,101,101,102,104,105, ... at times $\ldots, t, t+1, t+2, t+3, t+4, t+5, t+6, \ldots$

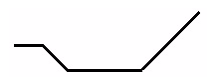

The data represents a valley at 101 at times $t+1$ (or $t+2$ or $t+3$ ). However, problems occur when the graphics tablet used produces a set of values like $\ldots, 102,101,102,101,102, \ldots$ which appear due to the hardware used and due to "false" pixels. False pixels occur when the line representing the pen's tip goes through the middle of the Cartesian grid representing the pixels of the signature capturing hardware (see Figure 2). If the written line goes exactly in between two pixels, then it is possible that the device will read the position as being sometimes on the left pixel and sometimes on the right. The pixel chosen by the device may be influenced by the way the signer is holding the pen, the pen's tilt or the pressure being applied by the user. So the data (say in the $y$ direction) may look something like this:

\section{$. ., 104,102,101,100,101,100,101,102,104$,}

Using the simple valley and peak detection algorithm, the above data returns a valley, followed by a peak, followed by another valley. Does this data have three turning points or does it have only a single valley? Now suppose the data looked like this:

$., 100,100,100,99,100,100,100, \ldots$

The simplest approach to removing the false turn-

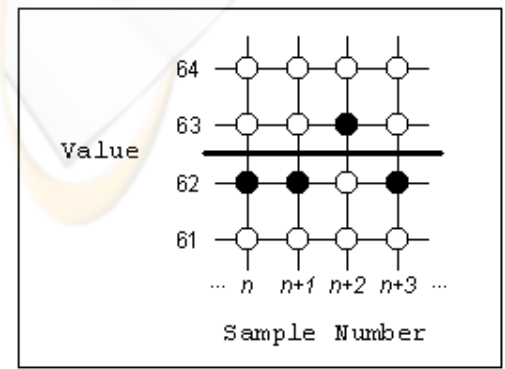

Figure 2: Example of how false turning points can occur using naive valley/peak detection algorithms.

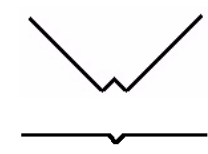

ing points involved removing valleys and peaks which had a depth or height less than some threshold. The valley depth (and similarly a peak) was measured in pixels and was calculated as the difference between the height of the valley and the height of the smallest of the immediately neighbouring peaks. Four experiments were conducted using this method for removal of valleys and peaks, which made use of the quality control technique. Valleys (and similarly peaks) were removed if a valley's depth was below a certain threshold value (measured in pixels). The four different experiments used four different thresholds none, two, three and four. The optimal overall error rate as well as the breakdown into FAR and FRR are presented for each of these thresholds in Table 1. As can be seen from this table, simple removal of small valleys and peaks clearly has a detrimental effect on verification accuracy. The only conclusion that can be drawn then is that valleys and peaks which have only a very small depth or height are important characteristics of the signing style.

Even though small valleys and peaks hold important detail relating to the signature's characteristic, something still needs to be done to remove "false" turning points. To do this, a different approach was adopted in which, put simply, at least two "down" events had to be followed by two "up" events for a valley to be detected. This was slightly different in that instead of considering the size of the valleys/peaks, the relative positions of the pen are being considered. That is, if the pen tip moves in a downward direction on two samples (without being interrupted by an "up"), then this is thought to be the start of a genuine valley. If the pen tip then moves back up for two samples (without being interrupted by a "down"), the genuine valley is completed and a valley is stored. See (McCabe and Trevathan, 2007) for a complete discussion of valley and peak detection algorithms developed in conjunction with this research.

\section{Performance of the Gupta and Joyce System}

Table 1: How removal of small valleys and peaks effects error rate.

\begin{tabular}{|cccc|}
\hline Threshold & FAR & FRR & Overall \\
\hline None & $4.0 \%$ & $3.1 \%$ & $7.1 \%$ \\
\hline 2 & $8.3 \%$ & $9.4 \%$ & $17.7 \%$ \\
\hline 3 & $9.2 \%$ & $10.3 \%$ & $19.6 \%$ \\
\hline 4 & $10.2 \%$ & $9.2 \%$ & $19.3 \%$ \\
\hline
\end{tabular}


Table 2: Initial results using the characters $A, B, C, D, F$.

\begin{tabular}{|cccccc|}
\hline$r$ & F-accept & G-reject & FAR & FRR & ER \\
\hline 0.0 & $45 / 325$ & $86 / 609$ & $13.8 \%$ & $14.1 \%$ & $27.9 \%$ \\
\hline 0.5 & $69 / 325$ & $41 / 609$ & $21.2 \%$ & $6.7 \%$ & $27.9 \%$ \\
\hline 1.0 & $107 / 325$ & $22 / 609$ & $32.9 \%$ & $3.6 \%$ & $36.5 \%$ \\
\hline 1.5 & $154 / 325$ & $12 / 609$ & $47.4 \%$ & $2.0 \%$ & $49.4 \%$ \\
\hline 2.0 & $188 / 325$ & $6 / 609$ & $57.8 \%$ & $1.0 \%$ & $58.8 \%$ \\
\hline
\end{tabular}

The (Gupta and Joyce, 1997a) technique was implemented following the experimental procedure described in their paper and the results presented in Table 2 were obtained. The $27.9 \%$ overall error rate was high because the technique was not capturing enough detail to discriminate well between signatures (only the ordering of turning points and the position and duration of pen-ups were captured). Table 2 shows that the FAR was the major problem, as it was too easy for a forger to produce the correct ordering of turning points, even though the actual signature may not be a very accurate forgery.

\section{Utilising Distance and Direction Characters}

Capturing the optimal level of signature detail is important to the overall success of the signature verification. Capturing too much detail leads to a high FRR since each genuine signature needs to be very similar to the reference signature(s), while capturing too little detail leads to a high FAR because it is easy for the forgeries to meet the minimal requirements. Table 1 shows that the FAR is high even for low threshold values. This suggests that the technique is capturing the shape well since most skilled forgeries reproduce the signature shape well. Furthermore, the technique is not capturing enough details other than the shape which could have reduced the skilled forgeries' FAR. Thus a modification of the technique that capture further signature details should improve performance.

Previously, only the ordering of the valleys and peaks in a signature were being captured and this approach allowed the skilled forgers to get the string representation similar to the genuine signatures, even though the forged signature was not an exceptionally good forgery. It was therefore decided to include more detail about the signature shape by including information about the stroke distances in the representation, which forces a forger (and genuine signer) to get not only the ordering of the valleys and peaks correct, but also the spacing between them. Additionally, the location and duration of "pen-up" occurrences were added to the signature representation.

How would the stroke distance between the turning point characters be represented? The distance itself may be computed in two different ways. It may be computed by finding the distance between the coordi-
Table 3: Error rates using the characters $A, B, C, D, P, I$, $J, K, L \ldots$ and using the time interval as the distance metric (distance intervals based on global characteristics).

\begin{tabular}{|cccccc|}
\hline$r$ & F-accept & G-reject & FAR & FRR & ER \\
\hline 0.0 & $48 / 325$ & $134 / 609$ & $14.8 \%$ & $22.0 \%$ & $36.8 \%$ \\
\hline 0.5 & $55 / 325$ & $109 / 609$ & $16.9 \%$ & $17.9 \%$ & $34.8 \%$ \\
\hline 1.0 & $60 / 325$ & $95 / 609$ & $18.5 \%$ & $15.6 \%$ & $34.1 \%$ \\
\hline 1.5 & $71 / 325$ & $67 / 609$ & $21.8 \%$ & $11.0 \%$ & $32.8 \%$ \\
\hline 2.0 & $77 / 325$ & $52 / 609$ & $23.7 \%$ & $8.5 \%$ & $32.2 \%$ \\
\hline
\end{tabular}

Table 4: Error rates using the characters $A, B, C, D, P, I$ $J, K, L \ldots$ and using the path length as the distance metric (based on global characteristics).

\begin{tabular}{|cccccc|}
\hline$r$ & F-accept & G-reject & FAR & FRR & ER \\
\hline 0.0 & $51 / 325$ & $135 / 609$ & $15.7 \%$ & $22.2 \%$ & $37.9 \%$ \\
\hline 0.5 & $57 / 325$ & $110 / 609$ & $17.5 \%$ & $18.1 \%$ & $35.6 \%$ \\
\hline 1.0 & $60 / 325$ & $96 / 609$ & $18.5 \%$ & $15.8 \%$ & $34.3 \%$ \\
\hline 1.5 & $74 / 325$ & $69 / 609$ & $22.8 \%$ & $11.3 \%$ & $34.1 \%$ \\
\hline 2.0 & $81 / 325$ & $55 / 609$ & $24.9 \%$ & $9.0 \%$ & $33.9 \%$ \\
\hline
\end{tabular}

nates of the two successive valleys/peaks. It may also be found distance in terms of the number of samples between successive valleys/peaks. Effectively then, the second alternative is determining the time lapsed between the turning point characters. A vector representing all of the distance values between the turning points of the signature image may then be obtained.

Using this approach, whatever distance technique is used, it is necessary to compute distance between vectors that are often of different sizes which is a difficult problem. One approach for comparing two vectors was to simply drop the smallest integers from the larger vector until the two lengths are the same. For example, suppose the following two vectors represent the distances between the turning point characters in two instances of a signature:

$$
\begin{aligned}
& 11862831216 \\
& 164322916
\end{aligned}
$$

If the smallest numbers from the longer vector are removed, the result is:

$$
\begin{aligned}
& 186283116 \\
& 164322916
\end{aligned}
$$

This idea has some merit, but difficulties can arise if the process leads to poor alignment of the vectors, therefore the approach was not investigated further. Instead, it was decided to map the integers to characters and include the distance information in the string representation itself.

To map the integers to characters, it was decided to first divide the distances into four classes. If the distance fell in the smallest quarter of the distances, the character $I$ was inserted into the string, next higher quarter distance were represented by a $J$, and simi- 
Table 5: Error rates using the characters $A, B, C, D, P, I$, $J, K, L \ldots$ and using the time interval as the distance metric (distance classes set automatically).

\begin{tabular}{|cccccc|}
\hline$r$ & F-accept & G-reject & FAR & FRR & ER \\
\hline 0.0 & $23 / 325$ & $100 / 609$ & $7.1 \%$ & $16.4 \%$ & $23.5 \%$ \\
\hline 0.5 & $33 / 325$ & $59 / 609$ & $10.2 \%$ & $9.7 \%$ & $19.8 \%$ \\
\hline 1.0 & $46 / 325$ & $35 / 609$ & $14.2 \%$ & $5.7 \%$ & $19.9 \%$ \\
\hline 1.5 & $64 / 325$ & $26 / 609$ & $19.7 \%$ & $4.3 \%$ & $24.0 \%$ \\
\hline 2.0 & $79 / 325$ & $13 / 609$ & $24.3 \%$ & $2.1 \%$ & $26.4 \%$ \\
\hline
\end{tabular}

larly the next higher distances were represented by $K$ and $L$. A pen-up character $P$ was also included in the string whenever the pen tip was not in contact with the writing tablet. Pen-up time was divided into small and large, where small pen-ups were represented by a single $P$ and large pen-ups represented as $P P$. The limits for each of the four distance classes were mutually calculated using the distance values for all users in the database. These limits were found by building a frequency vector for the distance values, and then dividing the distance values into four equal groups based on their magnitude, and assigning a symbol to each group. The limits for the distance classes were then used globally on the signature database to explore this approach's merit. The initial limits for the four classes for time and path length distances, and for pen-up occurrences were:

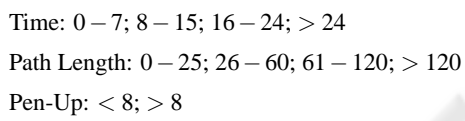

String representations of signatures were generated using the distance characters and the turning point characters. Performance evaluation of the technique using the time interval and the path length as the distance metric resulted in overall error rates of $32.2 \%$ and $33.9 \%$ respectively (see Table 3 and Table 4).

The above classes used in developing representations for the distance and the pen-up time were based on heuristics. Since the distances and the pen-up times tend to be quite different for different individuals, it was decided to select the classes based on typical distance values for each individual. To do this, five reference signatures for each individual were used, and all of the distance values in between turning point characters were obtained. These distance values were divided into four equal groups according to their magnitude and each group assigned a symbol. The small and large pen-up time intervals were also found in a similar fashion by dividing the pen-up time sizes into two equal groups according to magnitude. The performance was evaluated using the individualised intervals. The results obtained were an $19.8 \%$ over-
Table 6: Error rates using the characters $A, B, C, D, P, I$, $J, K, L \ldots$ and using the path length as the distance metric (distance classes set automatically).

\begin{tabular}{|cccccc|}
\hline$r$ & F-accept & G-reject & FAR & FRR & ER \\
\hline 0.0 & $25 / 325$ & $102 / 609$ & $7.7 \%$ & $16.7 \%$ & $24.4 \%$ \\
\hline 0.5 & $36 / 325$ & $58 / 609$ & $11.1 \%$ & $9.5 \%$ & $20.6 \%$ \\
\hline 1.0 & $48 / 325$ & $37 / 609$ & $14.8 \%$ & $6.1 \%$ & $20.9 \%$ \\
\hline 1.5 & $66 / 325$ & $27 / 609$ & $20.3 \%$ & $4.4 \%$ & $24.7 \%$ \\
\hline 2.0 & $81 / 325$ & $13 / 609$ & $24.9 \%$ & $2.1 \%$ & $27.0 \%$ \\
\hline
\end{tabular}

all error rate using the time interval as the distance metric and 20.6\% using the path length. Table 5 and Table 6 show that the best results are obtained when the threshold value is 0.5 . Although these results are much better than those in Table 3, the FAR is still high. From the results presented thus far, the time interval seems to be the superior distance metric of the two. Therefore we only concentrate on the time interval as the distance metric from this point on.

The results show that the FAR was still dominating the overall error rate indicating that shape was still the determining factor in the technique, since including the distance only improved the shape representation, but did not include any motion or dynamic information. One type of information that can be easily derived is the direction of pen motion at each moment. It was decided to incorporate details of the direction of the pen tip in between the valley and peak characters. To include pen tip direction, the motion direction was classified into four classes, corresponding to quadrants on a Cartesian grid. The character to include between the turning points $\alpha$, and the turning point $\beta$, can then be found by positioning $\alpha$ at the origin and determining which quadrant $\beta$ lies in.

Both the direction and the distance were included in the signature representation by using one symbol to represent both. To do this, for example, quadrant one and distance class one is represented by the character $E$, quadrant two and the distance class one is the character $F$ and so on, until there was a unique character for each of the sixteen direction/distance combinations. Table 7 presents the results of evaluating these two techniques. The results are much better with the minimum total error at $9.2 \%$.

An extension of the distance/direction approach involved attaching more weight to longer strokes. This was done by inserting not only the character appropriate for each stroke, but also the distance characteristic is considered, and the four distance classes (in order) are represented by the characters $W, X, Y$ and $Z$. If the distance between two valleys/peaks falls into the second largest class $Y$, then the three characters $W X Y$ would be inserted in between the valley/peak characters. This approach was considered 
Table 7: Error rates using the characters $A, B, C, D, P, E, F$, $G, H$ (direction components used).

\begin{tabular}{|cccccc|}
\hline$r$ & F-accept & G-reject & FAR & FRR & ER \\
\hline 0.0 & $3 / 325$ & $98 / 609$ & $0.9 \%$ & $16.1 \%$ & $17.0 \%$ \\
\hline 0.5 & $12 / 325$ & $43 / 609$ & $3.7 \%$ & $7.1 \%$ & $10.8 \%$ \\
\hline 1.0 & $25 / 325$ & $26 / 609$ & $7.7 \%$ & $4.3 \%$ & $12.0 \%$ \\
\hline 1.5 & $45 / 325$ & $19 / 609$ & $13.8 \%$ & $3.1 \%$ & $17.0 \%$ \\
\hline 2.0 & $60 / 325$ & $9 / 609$ & $18.5 \%$ & $1.5 \%$ & $19.9 \%$ \\
\hline 2.5 & $82 / 325$ & $5 / 609$ & $25.2 \%$ & $0.8 \%$ & $26.1 \%$ \\
\hline 3.0 & $96 / 325$ & $4 / 609$ & $29.5 \%$ & $0.7 \%$ & $30.2 \%$ \\
\hline
\end{tabular}

Table 8: Error rates using the characters $A, B, C, D, P, I, F$, $G, H, I, J, K, L$ (captures ordering, distance and direction).

\begin{tabular}{|cccccc|}
\hline$r$ & F-accept & G-reject & FAR & FRR & ER \\
\hline 0.0 & $1 / 325$ & $97 / 609$ & $0.3 \%$ & $15.9 \%$ & $16.2 \%$ \\
\hline 0.5 & $6 / 325$ & $45 / 609$ & $1.8 \%$ & $7.4 \%$ & $9.2 \%$ \\
\hline 1.0 & $15 / 325$ & $29 / 609$ & $4.6 \%$ & $4.8 \%$ & $9.4 \%$ \\
\hline 1.5 & $30 / 325$ & $19 / 609$ & $9.2 \%$ & $3.1 \%$ & $12.3 \%$ \\
\hline 2.0 & $47 / 325$ & $11 / 609$ & $14.5 \%$ & $1.8 \%$ & $16.3 \%$ \\
\hline 2.5 & $68 / 325$ & $6 / 609$ & $20.9 \%$ & $1.0 \%$ & $21.9 \%$ \\
\hline 3.0 & $76 / 325$ & $4 / 609$ & $23.4 \%$ & $0.7 \%$ & $24.1 \%$ \\
\hline
\end{tabular}

to have some advantage as (Dimauro et al, 1994) note that longer strokes are generally more difficult to forge. Accumulating the characters allows a level of variability for a genuine signer who only gets a long stroke slightly wrong. For example, suppose a reference signature contains the sequence WXYZ. In this situation, if the user formed the long stroke such that it was slightly shorter, the test signature would contain the sequence $W X Y$, so the mismatch would be quite small. Table 8 presents the results for the cumulative technique giving a best-case $9.2 \%$ overall error rate.

\section{Representing Stroke Length}

The approaches implemented thus far had involved increasing the level of detail which is captured by the signature representation. Table 8 shows that including more detail in the signature representation has improved the overall error rate significantly by lowering the FAR, but the FRR has increased as expected.

Table 9: Error rates obtained using the 'cumulative' technique.

\begin{tabular}{|cccccc|}
\hline$r$ & F-accept & G-reject & FAR & FRR & ER \\
\hline 0.0 & $1 / 325$ & $96 / 609$ & $0.3 \%$ & $15.8 \%$ & $16.1 \%$ \\
\hline 0.5 & $9 / 325$ & $46 / 609$ & $2.8 \%$ & $7.5 \%$ & $10.3 \%$ \\
\hline 1.0 & $15 / 325$ & $30 / 609$ & $4.6 \%$ & $4.9 \%$ & $9.5 \%$ \\
\hline 1.5 & $33 / 325$ & $19 / 609$ & $10.1 \%$ & $3.1 \%$ & $13.2 \%$ \\
\hline 2.0 & $46 / 325$ & $12 / 609$ & $14.2 \%$ & $1.9 \%$ & $16.1 \%$ \\
\hline 2.5 & $64 / 325$ & $7 / 609$ & $19.7 \%$ & $1.1 \%$ & $20.8 \%$ \\
\hline 3.0 & $77 / 325$ & $4 / 609$ & $23.4 \%$ & $0.7 \%$ & $24.1 \%$ \\
\hline
\end{tabular}

To improve the error rates further, it is necessary to study the representation and improve it if possible. It should be noted that including the direction information in the signature representation is not necessary since the direction of pen motion is implied by the peak-valley representation. For example, if a $x$-peak is followed by a $y$-valley, then the direction of pen motion between the two must be southwest.

Therefore it was decided to refine the signature representation yet again. Two changes were introduced, firstly the direction information was removed since the direction is implicit in the peak-valley representation as noted above. Also, the representation of the distance between each successive turning points was changed. Using single characters to represent different distance magnitudes was perhaps leading to problems since the signatures of each individual have variations, and if the two corresponding stroke distances were somewhat different they could well be represented by different symbols leading to a mismatch when the signature representations are compared. Therefore a new representation was developed which retains the valley and peak characters, but instead of using a single character to represent each distance (or direction or both), multiple identical characters are used to represent it. The number of characters used is proportional to the magnitude of the distance. The strength of this representation is that if two corresponding stroke distances are somewhat different in two signatures, the corresponding representation would not be very different, it might only differ by a single character in a string of characters that represent those two corresponding distances. Let the distance be represented by a number of $T$ characters in between the turning point characters. Using multiple characters also captures pen tip velocity to an extent, as the number of $T$ characters in between the segment will be less if the pen is being moved quickly.

It is possible to insert a $T$ character for each signature sample point, but this can result in strings well over one thousand characters in length. This not only results in string matching requiring significant resources, but leads to an ineffective signature representation since the representation mostly consists of the $T$ characters, and the fifty or sixty turning point characters do not have a significant influence on the results of signature comparisons. It is essential that the number of $T$ characters included to represent the stroke distances is not much more than the number of turning point characters. One approach is to insert a $T$ character for every $n$ signature sample points (or time units) where $n$ perhaps has a range of 100 to 300). The characters which are now included are the four turning point characters $A, B, C, D$, the pen-up char- 
Table 10: Error rates using the characters $A, B, C, D, P, T$.

\begin{tabular}{|cccccc|}
\hline$r$ & F-accept & G-reject & FAR & FRR & ER \\
\hline 0.0 & $0 / 325$ & $117 / 609$ & $0.0 \%$ & $19.2 \%$ & $19.2 \%$ \\
\hline 1.0 & $9 / 325$ & $40 / 609$ & $2.7 \%$ & $6.5 \%$ & $9.2 \%$ \\
\hline 2.0 & $17 / 325$ & $19 / 609$ & $5.2 \%$ & $3.1 \%$ & $8.3 \%$ \\
\hline 3.0 & $24 / 325$ & $13 / 609$ & $7.4 \%$ & $2.1 \%$ & $9.5 \%$ \\
\hline 4.0 & $36 / 325$ & $7 / 609$ & $11.0 \%$ & $1.2 \%$ & $13.2 \%$ \\
\hline
\end{tabular}

acter $P$, and a new character $T$ representing the time spacing between the turning point characters.

The signature representation now essentially represents a signature as a description similar to the following: "The pen moved northwest for $100 \mathrm{~ms}$ then southwest for $150 \mathrm{~ms}$ then southeast for $75 \mathrm{~ms}$... etc." This appears to be a flexible representation which still captures a significant level of detail.

The results in this section were obtained by inserting a $T$ character for every $n$ time units. This places a large emphasis on correct spacing of turning points, but at the same time the direction information is represented in the ordering of the turning points themselves. The representation also captures pen tip velocity to an extent, as the number of $T$ characters in between a segment will be less if the pen is being moved quickly. Six sets of results were obtained corresponding to different values of $n$ ranging from $n=2$ to $n=8$. However, only the results which were most attractive (those which used an $n$ value of four) are presented (Table 10). The total error rate is now $8.3 \%$ (at a threshold of 2.0) compared to $9.2 \%$ in Table 8 .

\section{Controlling Reference Signature Quality}

A HSV system's reliability depends on the quality of the reference signatures. It is assumed that these signatures provide an accurate indication of how a person's signatures vary. A HSV system is going to have difficulties if the sample signatures are very similar, i.e., they show less variation than the person's signatures actually do. The system will suffer from a high FRR but will have a low FAR. Also, if the sample signatures show more variation than is normal for the person, then the system will lead to higher FAR but lower FRR. If possible, a HSV system should try to protect itself from such problems.

Heuristics have been developed to check the reference signature quality, and to determine if the level of variability in the reference signatures is unusually high or low, and correcting it if it is. The variability of the reference signatures can be judged by the ratio of the standard deviation to the mean of the distances between reference signatures. If this ratio is too small, then the reference signatures are unusually similar. If the ratio is large, the reference signatures are unusually different. It was decided to place a lower limit of
1.75 on this ratio and if the ratio was found to be below 1.75 , it was assumed that the reference signatures were too similar, and the reference standard deviation was adjusted up to 1.75 times the reference mean. In experimentation a limit of 1.5 was also considered, but the limit of 1.75 produced superior results. Imposing the lower limit on the ratio should reduce the FRR although it might increase the FAR somewhat.

The decision on reference signatures being too different was not based on the ratio of the standard deviation to the mean of the distances between the reference signatures. Instead, the ratio of the mean to the distances between the reference signature representations to the average length of that person's signature representations was examined. If this ratio was large, then it was assumed that the reference signatures had too much variation. It was decided to impose an upper limit of 0.25 on this ratio. If the ratio was greater than 0.25 , then the reference mean was reduced to 0.25 times the average length of the reference signatures' representations. Values of 0.2 and 0.225 were also considered in experimentation, but performance evaluation indicated that the 0.25 limit was superior. This approach would be expected to reduce the FAR while still allowing genuine signers a large degree of variability so as not to significantly raise the FRR.

Modifying the last technique that used multiple characters to represent distance and imposing the above upper bound and lower limits on the two ratios, the technique was tested again and the detailed results are presented in Table 11. A minimum 4.1\% total error rate is obtained which is a very significant improvement on the results without the limits. The technique is now quite reliable with a $1.8 \%$ FRR and a $2.3 \%$ FRR. Smaller FAR or FRR is possible, but the total error rates are then higher than the minimum $4.1 \%$ total error rate.

It is possible to investigate other modifications of the techniques that have been investigated. In a practical system, it may be sufficient to just print a warning when a user provides the reference signatures if the variability of the signatures is unusually high or low. There is then the option of requesting a new set of reference signatures.

\section{Path Length and Elapsed Time}

A slightly different extension to the earlier technique that used only distance characters involved using both the path length and the elapsed time in the signature representation. The approach's purpose was to incorporate both the physical spacing and the elapsed time in between turning points, thereby capturing a notion of pen tip velocity. These features were incorporated by inserting the appropriate number of $T$ characters firstly, and then inserting a number 
Table 11: Error rates obtained after adjustment of variation.

\begin{tabular}{|cccccc|}
\hline$r$ & F-accept & G-reject & FAR & FRR & ER \\
\hline 0.0 & $0 / 325$ & $141 / 609$ & $0.0 \%$ & $23.2 \%$ & $23.2 \%$ \\
\hline 0.5 & $0 / 325$ & $69 / 609$ & $0.0 \%$ & $11.3 \%$ & $11.3 \%$ \\
\hline 1.0 & $3 / 325$ & $27 / 609$ & $0.9 \%$ & $4.4 \%$ & $5.4 \%$ \\
\hline 1.5 & $6 / 325$ & $14 / 609$ & $1.8 \%$ & $2.3 \%$ & $4.1 \%$ \\
\hline 2.0 & $8 / 325$ & $13 / 609$ & $2.5 \%$ & $2.1 \%$ & $4.6 \%$ \\
\hline 2.5 & $23 / 325$ & $11 / 609$ & $7.1 \%$ & $1.8 \%$ & $8.9 \%$ \\
\hline 3.0 & $37 / 325$ & $8 / 609$ & $11.4 \%$ & $1.3 \%$ & $12.7 \%$ \\
\hline
\end{tabular}

Table 12: Error rates using path length and elapsed time.

\begin{tabular}{|cccccc|}
\hline$r$ & F-accept & G-reject & FAR & FRR & ER \\
\hline 0.0 & $0 / 325$ & $140 / 609$ & $0.0 \%$ & $23.0 \%$ & $23.0 \%$ \\
\hline 0.5 & $1 / 325$ & $72 / 609$ & $0.3 \%$ & $11.8 \%$ & $12.1 \%$ \\
\hline 1.0 & $4 / 325$ & $29 / 609$ & $1.2 \%$ & $4.7 \%$ & $5.9 \%$ \\
\hline 1.5 & $6 / 325$ & $15 / 609$ & $1.8 \%$ & $2.5 \%$ & $4.3 \%$ \\
\hline 2.0 & $8 / 325$ & $14 / 609$ & $2.4 \%$ & $2.3 \%$ & $4.7 \%$ \\
\hline 2.5 & $23 / 325$ & $12 / 609$ & $7.1 \%$ & $2.9 \%$ & $9.1 \%$ \\
\hline 3.0 & $39 / 325$ & $9 / 609$ & $12.0 \%$ & $1.5 \%$ & $13.5 \%$ \\
\hline
\end{tabular}

of $S$ characters (one $S$ for every $m$ pixels). Table 12 presents the results using this technique where the optimal uses a value of thirty for $m$ and one $T$ for every four time units. The error rates produced using this technique were slightly higher than those found in Table 11 , including an optimal $4.3 \%$ overall error rate.

\section{Zero Effort FAR}

Tests were performed using other users' genuine signatures as zero-effort forgeries. This test's purpose was to determine how effective the HSV system was if the forger had no knowledge of the genuine user's signing style, and just signed his or her own signature. The FAR for such a test should be much smaller than for skilled forgeries, and ideally should be close to zero, although it is known that for some feature-based techniques the zero-effort FAR is not low. The total number of zero-effort forgeries was 71282, since for each signature in the database, every signature for $e v$ ery other user is a zero-effort forgery. The optimal result for zero-effort forgeries will always be at $r=0.0$ since the FRR is not a problem. At this threshold the overall zero-effort FAR is $0.0 \%$. However, it is more realistic to use the same global threshold that was optimal for the skilled FAR tests in Table 11. When this threshold ( $r=1.5$ ) was used, the zero-effort FAR is $1.4 \%$, which was quite low.

\section{Fewer Reference Signatures}

The experiments performed so far have involved the use of five reference signatures in order to establish the normal variability level for a particular user. Additional experiments were undertaken to explore the possibility of using fewer reference signatures, perhaps three or even just one. When only a single reference signature was used, no value for variability could be obtained, so the reference mean was set to 0.25 times the length of the reference signature character string and the reference standard deviation was set to 1.75 times the reference mean. The overall error rates using three and one reference signatures remained quite low at $8.4 \%$ and $12.8 \%$ respectively. Few techniques in literature report results this accurate when such a limited reference set is used.

\section{Individual Thresholds}

The results reported in this paper have been obtained using the same threshold for all users in each test. The results would improve significantly if optimal threshold values were used for each individual. Many studies reported in literature use individual thresholds, although none of them reports how an individual threshold can be computed given only the reference signatures. Generally, all studies using individual thresholds arrive at locally optimal thresholds by using the threshold for the lowest error rate for each user individually, and then summing these error rates to determine the overall error rate. If this approach is used the total error rate reduces from $4.1 \%$ to $1.3 \%$, which consists of a $1.3 \%$ FRR and $0 \%$ FAR. The total number of misclassified signatures being just eight. Additionally, only four users contribute to this misclassification, meaning that 55 users (i.e., $93 \%$ of the 59 users) would have a $0 \%$ overall error rate if locally optimal thresholds were used. Clearly, this shows the technique is highly reliable.

\section{CONCLUSIONS}

The results presented in this paper show that the proposed HSV system is comparable to the highest quality signature verifiers presented in literature. The features which have led to this success include:

The use of a string to represent the signature - this allows existing string matching algorithms to be used to determine distances between signatures. Furthermore, this makes the signature verifier quite tolerant to variations in genuine signatures. For example, in the signature database there was one individual who sometimes signed using his middle initial, while other times signed without his middle initial. When these signatures are represented as strings, the only real difference are the characters in the middle of the signature. The signature verifier handles the situation well, reporting a $20 \%$ FRR and a $0 \%$ FAR using the global optimum threshold. Few other HSV systems perform this well in such an extreme case. 
Reference signature quality checking - This is uncommon in literature. It determines if the reference signature variability is too high/low, and if so, adjusting the reference mean and standard deviation.

Use of a small number of reference signatures Using five reference signatures led to a $4.1 \%$ overall error rate. Results using three and even one reference signature were also quite attractive (as low as $8.7 \%$ using three and $12.5 \%$ using just one) when compared to similar experiments in literature.

The overall results used a single globally optimal threshold value (i.e., the threshold value was the same for each user). By choosing a locally optimal threshold for each individual the total error rate drops to just $1.3 \%$ FRR and $0 \%$ FAR.

\section{REFERENCES}

Dimauro, G., Impedovo, S., and Pirlo, G. (1994). Component-Oriented Algorithms for Signature Verification. International Journal of Pattern Recognition and Artificial Intelligence. Vol 8, No 3, 771-793.

Gupta, G.K., and Joyce, R.C. (1997a). A Sudy of Shape in Dynamic Handwritten Signature Verification. Submitted for publication.

Gupta, G.K., and Joyce, R.C. (1997b). A Sudy of Some Pen Motion Features in Dynamic Handwritten Signature Verification. Submitted for publication.

McCabe, A. (2000). Markov Modelling of Simple Directional Features for Effective and Efficient Handwriting Verification. R. Mizoguchi and J. Slaney (Eds.): PRICAI 2000, LNAI 1886, 801(1-12).

McCabe, A. and Trevathan, J. (2007). Pre-processing time dependent signals in a noisy environment, In Proceedings of the $4^{\text {th }}$ International Conference on Information Technology - New Generations, 393-397.

Nelson, W., Turin, W. and Hastie, T. (1994). Statistical Methods for On-line Signature Verification., In International Journal of Pattern Recognition and Artificial Intelligence, vol. 8, No. 3, 749-770.

Trevathan, J. and McCabe, A. (2005). Remote Handwritten Signature Authentication, In Proceedings of the $2^{\text {nd }}$ International Conference on e-Businees and Telecommunications Engineers, 335-339.

Wagner, R. A. and Fischer, M. J. (1974). The String-toString Correction Problem. Journal of the ACM. Vol 21, No 8, 168-173. 